\title{
Contribution à l'étude de la salinisation de la nappe côtière de sahel El Haouzia région d'El Jadida au Maroc
}

\section{Nabil MDIKER ${ }^{1 *}$, Abderrahim El ACHHEB' ${ }^{\prime}$, Abdenbi MANDOUR ${ }^{2}$, Abdelkader YOUNSI ', Soufiane El MALIKI' et B Khadija OUTEYEB'}

'Laboratoire Géosciences et Techniques de l'Environnement,Département de Géologie, Faculté des Sciences .Route Ben Maachou,24000 El Jadida, Maroc.

${ }^{2}$ Laboratoire Géologie Appliquée, Hydrogéologie et Environnement,Département de Géologie Faculté des Sciences Route sidi Maafa,60000. Oujda, Maroc.

\footnotetext{
*Correspondance,courriel :mdikernabil@yahoo.fr
}

\section{Résumé}

La nappe côtière du Sahel d'El Haouzia circulent soit dans les calcaires fissurés du Cénomanien (partie amont), soit dans les formations détritiques du Plioquaternaire (partie avale). Ce sont des formations perméables favorisant le transit des eaux d'infiltration à forte charge saline, vers la nappe d'eau souterraine, circulant généralement à de faibles de la surface. La conductivité électrique moyenne des eaux souterraines étudiées montre des variations importantes, de $1,7 \mathrm{mS} / \mathrm{cm}$ (zone interne) à $7 \mathrm{mS} / \mathrm{cm}$. En effet, elle accuse des valeurs de plus en plus importantes en direction de la mer, avec un gradient d'augmentation important dans les premiers kilomètres du rivage. La minéralisation totale est déterminée principalement par les ions chlorures et sodium qui montrent une corrélation positive avec la conductivité électrique.

La combinaison des outils, géologiques, hydrogéologiques, piézométriques et hydrochimiques a montré que les fortes salinités des eaux souterraines sont liées au phénomène d'intrusion marine (dans les premiers kilomètres de la côte), à celui du lessivage de la roche réservoir et de l'infiltration des eaux de retour. Ces méthodes d'étude ont montré une meilleure adaptation avec notre système aquifère côtier.

Mots-clés : Nappe côtière, salinité, intrusion marine, hydrochimie, Sahel EI Haouzia, Maroc. 


\section{Abstract \\ Contribution for study of the salinization of costal aquifer of El Haouzia sahel region El Jadida, Morocco}

The coastal aquifer of El Haouzia Sahel circulate either in Cenomanian limestones (crack permeability) in the south east part or in the sandy calcareous formations of Plioquaternary in downstream part. These aquifer formations caracterized by important permeabilities and a small depth of piezometric level below a sand-clayey soil, are regarded a priori as sensitive to saline pollution.

The electric conductivity average of groundwaters studied shows important variations of $1.7 \mathrm{mS} / \mathrm{cm}$ (south east part) to $7 \mathrm{mS} / \mathrm{cm}$ (coastal areas). Indeed it shows increasingly important values in direction of the sea, with an important gradient of the increase in the first kilometers from the coast. The total mineralization is determined mainly by the sodium and chlorides ions which show a positive correlation with the electric conductivity.

The combination of geological, hydrogeological and geochemical methods shows that the high salinities of groundwaters are related to: seawater intrusion (in the first kilometers of the coast), aquifer formation lixiviation, infiltration of water of return and a possible trapping of old seawater. These methods of study show a better adaptation with our coastal aquifer system.

\section{Keywords : Coastal aquifer, salinity, marine intrusion, geochemistry, El Haouzia Sahel, Morocco.}

\section{Introduction}

Les aquifères côtiers constituent des ressources en eau importantes dans les zones littorales où l'on observe des fortes densités démographiques et une intense activité agricole. Ce sont des zones où la demande en eau est de plus en plus croissante. Cela est favorisé par la faible profondeur des nappes d'eau souterraine dans ces zones côtières. Cependant, l'exploitation de ces nappes pose des problèmes complexes car elle associe la notion des réserves des eaux souterraines à celle de leur qualité [1-5] Le Sahel El Haouzia fait partie de ces zones côtières, où l'on observe un essor démographique important et une forte activité agricole. Les seules ressources en eau pour l'alimentation de la population rurale et pour l'irrigation des cultures, correspondent aux eaux souterraines. Ces eaux circulent à des profondeurs moyennes de $15 \mathrm{~m}$. 
Le but de notre travail est de contribuer à comprendre les causes de salinisation des eaux souterraines du Sahel d'El Haouzia en utilisant et en combinant plusieurs méthodes d'étude : géochimie des eaux, piézométrie, géologie, hydrogéologie.

\section{Matériel et méthodes}

\section{2-1. Présentation du site d'étude}

\section{2-1-1. Présentation géographique}

La région étudiée fait partie du domaine mésétien du Maroc occidental (Figure I) compris entre les latitudes $33^{\circ} 18^{\prime}, 33^{\circ} 05^{\prime}$ et les longitudes $8^{\circ} 30^{\prime}, 8^{\circ} 20^{\prime}$ à l'echelle du globe. Elle est limitée au Nord par l'océan atlantique, au Sud par les Doukkala, à l'Est par l'Oued Oum Errabia et à l'Ouest par le plateau d'El Jadida et s'étend sur une superficie d'environ $500 \mathrm{Km}^{2}$.

L'élevage et l'agriculture représentent les activités les plus pratiquées par la population de la région étudiée. Ce sont des activités basées sur les ressources en eau souterraine, circulant à de faibles profondeurs de la surface. Ce qui fait de ces zones, des secteurs à priori sensibles à la contamination.

\section{2-1-2. Présentation géologique}

Le secteur d'étude (Figure \) présente deux entités géologiques, bien différenciées, le socle primaire plissé sur lequel repose des terrains postérieurs formant la couverture d'âge secondaire, tertiaire et quaternaire $[6,7]$.

\section{Géologie superficielle de la zone d'étude}

\section{2-1-2-1. Crétacé inférieur ou moyen}

Le Crétacé correspond à une période de transgression marine généralisée sur toute la région. II constitue une épaisse série de marnes vertes ou bleues qui peut atteindre $200 \mathrm{~m}$ environ, avec des bancs marno-calcaires. Le gypse tient une place importante dans cette série, soit en bancs soit en masses. Ces dépôts Crétacés sont extrêmement importants pour l'hydrogéologie puisqu'ils forment le substratum des terrains cénomaniens et plioquaternaires, principaux aquifères de la zone d'étude. 
L'absence de fossiles ne permet pas de dater cette formation. Leur position entre le Néocomien (argiles rouges supérieures) et le Cénomanien autorise l'appellation vague de ॥ Crétacé inférieur ou moyen ॥. [7-9].

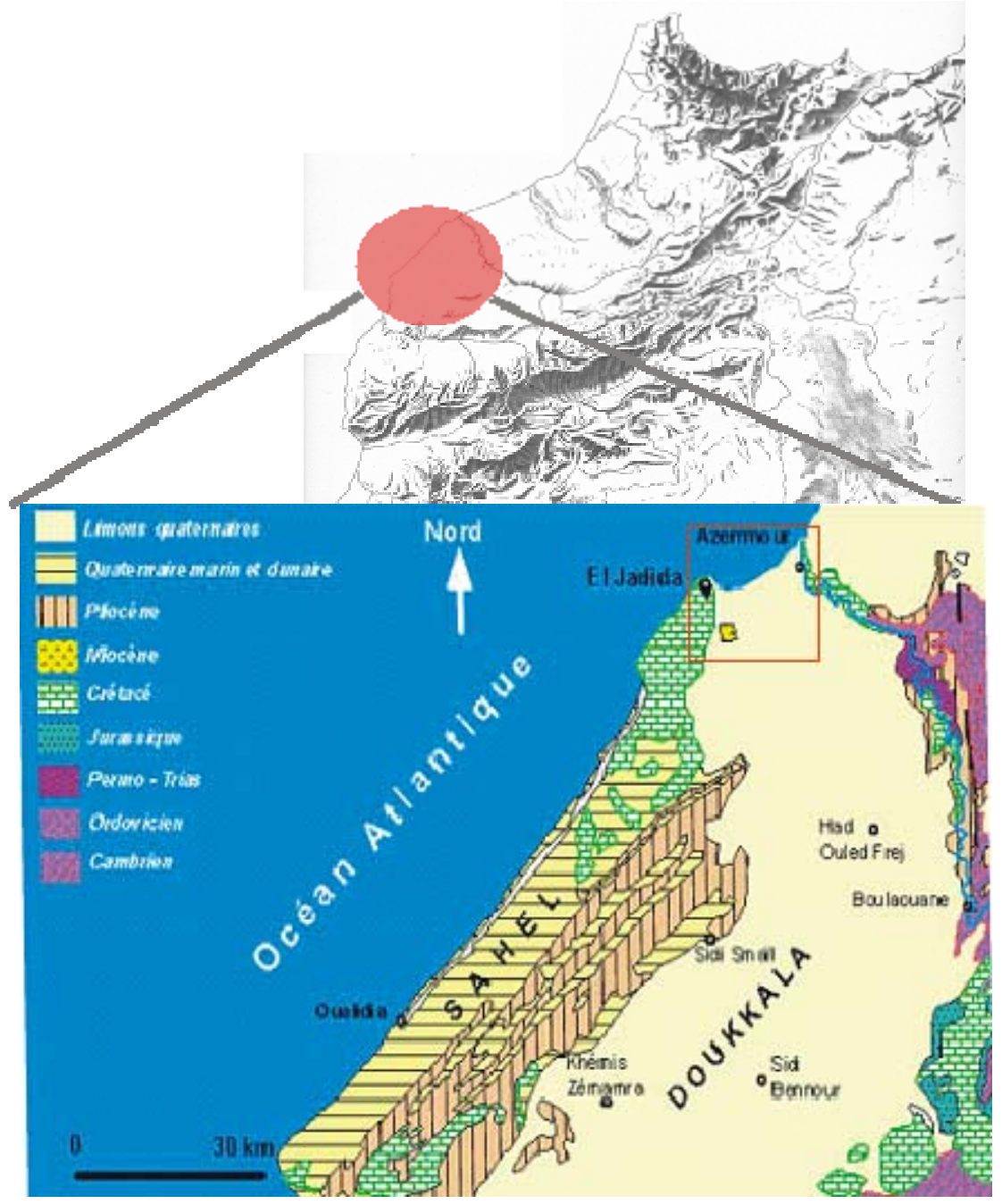

$\square$ Limite de la zone d'Etude

Figure 1 : Situation géographique de la région étudiée 


\section{2-1-2-2. Cénomanien}

Au Cénomanien, une nouvelle transgression a repris, cette transgression a donné naissance à des dépôts calcaires et marno-calcaires. Ces formations affleurent dans la vallée de l'Oued Oum Rbiaa, au sud et au sud ouest d'El Jadida. Le Cénomanien représente l'aquifère le plus étendu dans la zone d'étude [7-9].

\section{2-1-2-3. Plioquaternaire}

Au Plioquaternaire, une nouvelle transgression marine plus importante a recouvert presque toute la région, elle est arrivée jusqu'aux pieds des Rehamna. La régression qui a suivie, a été marquée par la formation des dunes côtières formées par le même matériel, ce qui a rendu difficile de distinguer entre les dépôts marins et dunaires. Les différents faciès qui constituent les dépôts plioquaternaires, sont :

*conglomérats grossiers qui caractérisent l'ancienne embouchure de l'0um Rabiaa ;

*calcaires détritiques jaunes contenant des débris de coquilles très poreux ;

*sables jaunes grossiers.

L'épaisseur des dépôts plioquaternaires est variable, de 10 à $60 \mathrm{~m}$ dans certaines zones [8-11].

\section{2-1-3. Présentation hydrogéologique du Sahel El Haouzia}

Elle a révélé deux faciès lithologiques capables de jouer le rôle d'un bon aquifère sur un substratum marneux du Crétacé moyen ou inférieur. Ces deux formations aquifères se présentent comme suit :

* Les grès et les calcaires lumachelliques du Plioquaternaire qui sont caractérisés par une porosité et une bonne perméabilité d'interstice, mais leur extension latérale reste limitée pour former un aquifère généralisé sur toute la zone. C'est une formation aquifère exploitée principalement dans la bande côtière.

* Les calcaires fisuré et les calcaires marneux du Cénomanien qui représentent l'aquifère le plus étendu du Sahel El Houzia. Cet aquifère est caractérisé par une perméabilité moyenne de fissures et par des débits d'eau relativement importants.

Ces deux aquifères plioquaternaire et cénomanien sont superposés et sont en communication hydraulique verticale.

L'alimentation du système aquifère est assurée principalement par l'infiltration et la percolation des eaux météoriques et aussi par les apports souterrains latéraux provenant de la plaine des Doukkala [9]. 


\section{2-2. Méthodes}

Dans le cadre de ce travail, nous avons procédé à un maillage régulier de la zone étudiée et nous avons sélectionné un réseau représentatif de 24 puits, dont les résultats seront analysés dans ce travail (Figure 2). Ces puits ont fait l'objet de campagne de relevés piézométriques et de mesures des paramètres physiques de l'eau in situ (conductivité, $\mathrm{pH}$,température, $\mathrm{O}_{2}$ dissou) ainsi de prendre des échantillons d'eau dans des flacons de polythlènes a pour but d'analyses chimiques au laboratoire. Tous ces puits ont été reportés sur carte topographique et repérés grâce au GPS. Les résultats obtenus nous ont permis de dresser certain nombre de cartes (piézométrique, et certain éléménts chimiques).

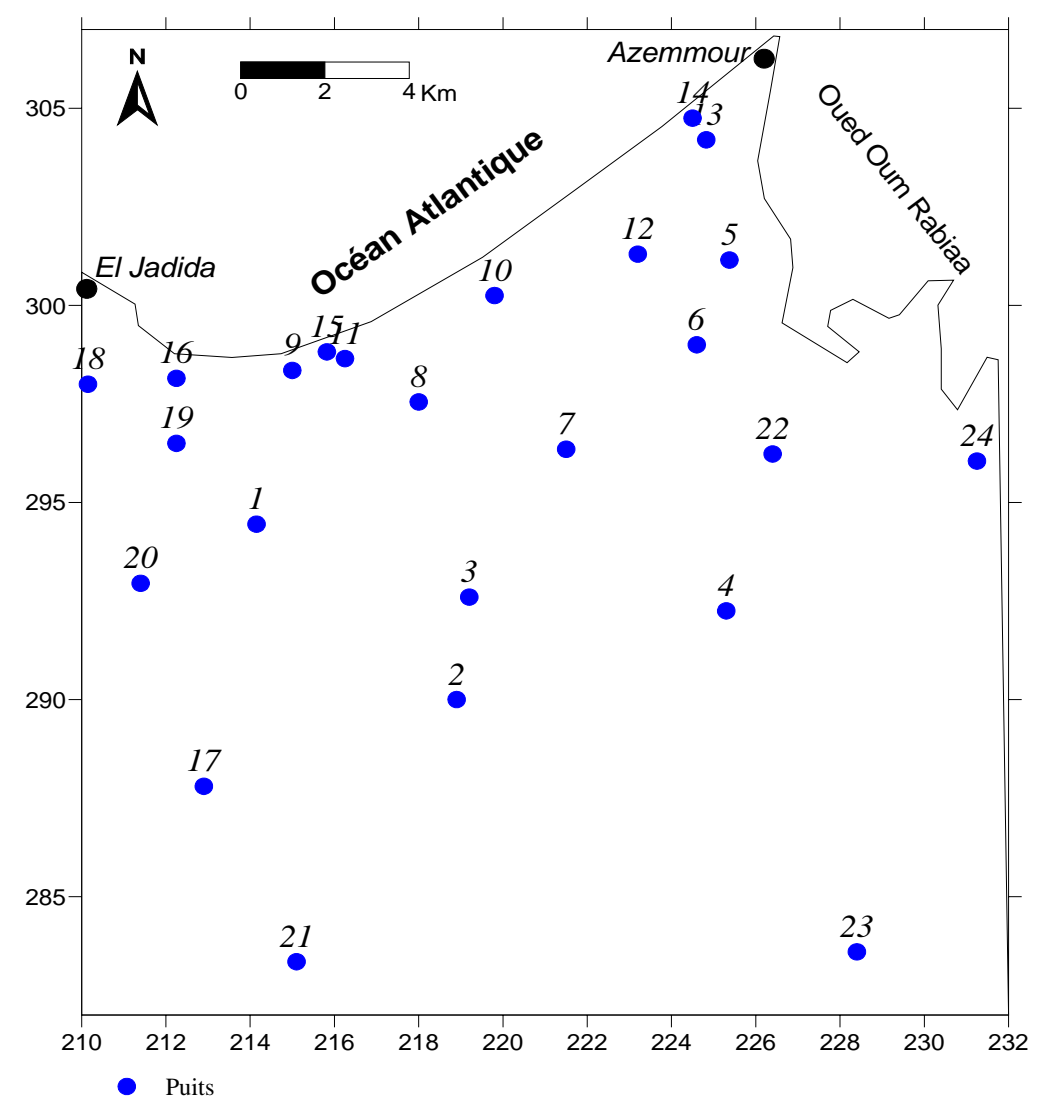

Figure 2 : Répatition géographique des points de mesure 


\section{Résultats}

\section{3-1. Carte piezomètrique}

La (Figure 3) représente une carte piézométrique de la période des basses eaux (Août 2005) et elle va nous permettre de caractériser les directions et les sens d'écoulement de la nappe ainsi que les zones de drainage et l'évolution du gradient hydraulique de la nappe. En effet, l'écoulement général de la nappe se fait du Sud vers le Nord, vers l'océan (exutoire principal de l'aquifère). Cet état piézométrique montre l'existence de deux zones:

- Une zone où les isopièzes sont espacées qui correspond à l'aval de la nappe et qui est caractérisé par un gradient hydraulique faible de l'aquifère. Dans cette zone, la nappe circule dans les terrains plioquaternaires.

- Une zone à l'amont où les isopièzes sont resserrées et les gradients hydrauliques sont élevés. Ce qui traduit que dans cette zone où l'aquifère est bien développé dans les formations fissurées du Cénomanien.

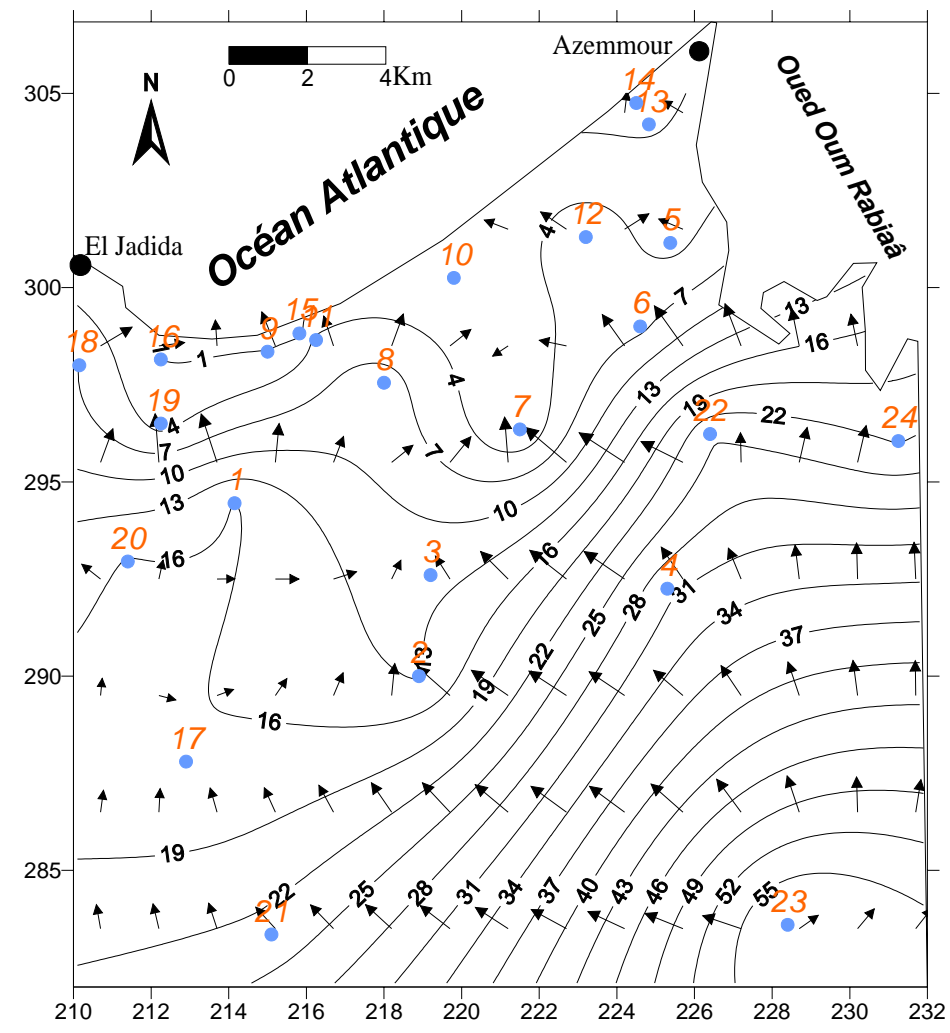

Figure 3 : Carte piézometrique de Sahel El Haouzia (Août 2005) 


\section{3-2. Etude géochimique des eaux}

Le même réseau de puits (Figure 2) a fait l'objet de campagnes d'échantillonnage d'eau Août 2005. Sur le terrain, nous avons mesuré les différents paramètres physiques : température, pH, conductivité électrique, oxygène dissous.

Les échantillons pris directement des points d'eaux sont conservés dans une glacière et emmenés au laboratoire pour analyses des paramètres chimiques majeurs suivants: $\mathrm{Na}^{+}, \mathrm{K}^{+}, \mathrm{Ca}^{2+}, \mathrm{Mg}^{2+}, \mathrm{Cl}^{-}, \mathrm{HCO}_{3}{ }^{-}, \mathrm{SO}_{4}{ }^{2-}, \mathrm{NO}_{3}$.

\section{3-2-1. Conductivité électrique}

Les mesures obtenues in situ (Août 2005) oscillent entre une valeur minimale de 1.44 $\mathrm{mS} / \mathrm{cm}$ et une valeur maximale de $7.20 \mathrm{mS} / \mathrm{cm}$, avec une moyenne de l'ordre 3.43 $\mathrm{mS} / \mathrm{cm}$ et un écart-type de 1.30 .

Et pour mieux comprendre et visualiser la minéralisation des eaux de la nappe du Sahel d'El Haouzia, nous avons pu dresser la carte de distribution spatiale de conductivités électriques sur l'ensemble du secteur de travail.

L'analyse de la carte de la (Figure 4) nous a permis de distinguer deux zones bien marquées:

-La partie amont où les valeurs de conductivité électrique sont faibles et augmentent au fur et à mesure que l'on se rapproche de l'Océan.

-Le secteur côtier de la zone étudiée où l'on observe des plages de conductivité électrique plus élevées, supérieures à $3.5 \mathrm{mS} / \mathrm{cm}$. Les valeurs peuvent atteindre 7.2 $\mathrm{mS} / \mathrm{cm}$ au Nord Est. Cette forte minéralisation des secteurs côtiers peut être expliquée par le phénomène de l'intrusion marine vers la nappe côtière à la suite d'un abaissement du niveau piézométrique. Cet abaissement est dô aux pompages intensifs et fréquents dans la zone étudiée qui ne dispose que des eaux souterraines pour son alimentation quotidienne et pour l'irrigation. Ce phénomène d'intrusion marine semble être favorisé par la faible épaisseur aquifère saturée (faible gradient hydraulique), par le plongement sous le zéro marin du substratum marneux du Crétacé moyen ou inférieur et principalement par la surexploitation de la nappe côtière, surtout pendant la période sèche.

D'autres sources potentielles contribuant aux fortes salinités peuvent être citées, en l'occurrence les aérosols marins, l'utilisation excessive des sels agricoles, l'existence d'une éventuelle paléosalinité piégée,... En effet, les aérosols contiennent des cristaux de sels (principalement le $\mathrm{NaCl}$ ) qui se déposent sur les sols proches de la 
mer (jusqu'à $15 \mathrm{~km}$ ). Leur lessivage par les eaux pluviales ou d'irrigation les entraîne vers la nappe proche de la surface, provoquant ainsi sa minéralisation.

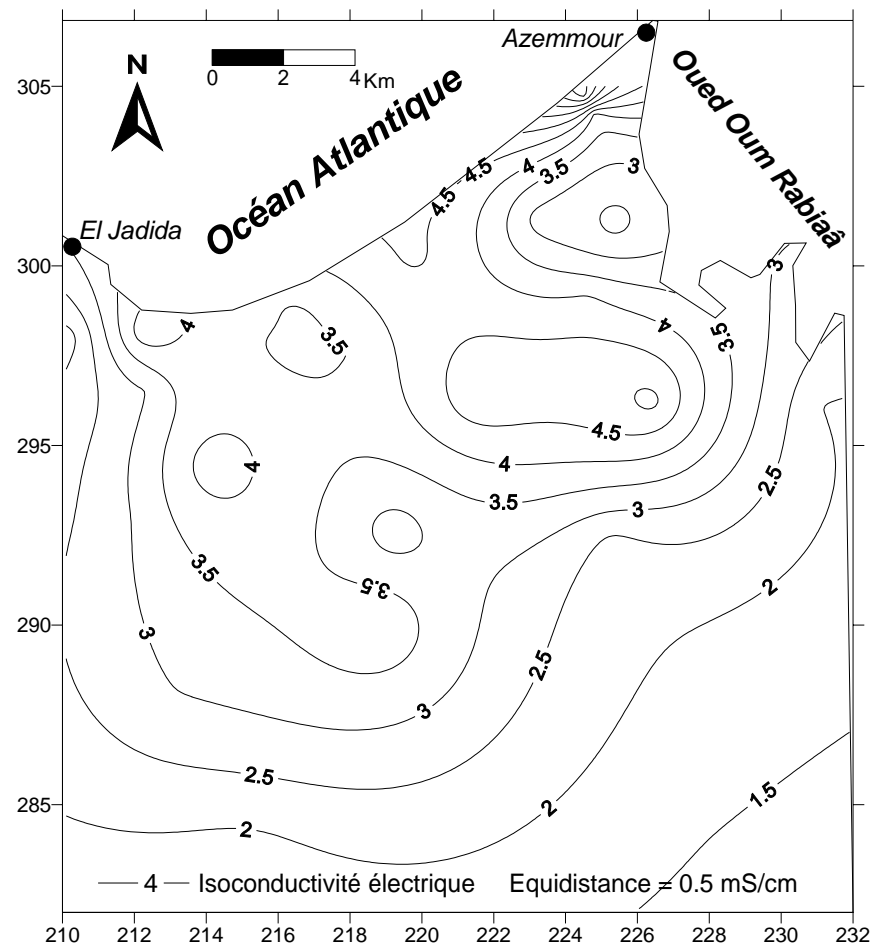

Figure 4 : Carte de conductivité électrique de Sahel El Haouzia (Août, 2005)

\section{3-2-2. Les chlorures et sodium}

Les teneurs en chlorures et sodium dans les eaux montrent une variation spatiale similaire à celle de la conductivité électrique. Celle-ci est déterminée principalement par ces deux ions, d'où un faciès chloruré sodique dominant.

Cette corrélation positive entre la conductivité électrique (CE) et les ions chlorures et sodium a été démontrée par le (Tableav 1). La différence entre ces deux coefficients peut être expliquée par le fait que les ions sodium subissent un transport non conservatif (échanges cationiques avec le sol), alors que les chlorures ne subissent aucune transformation pendant leur transport. l'Analyse en Composantes Principales (ACP) des différentes données physico-chimiques obtenues sur les puits suivis plaide aussi en faveur de cette corrélation positive entre la conductivité et les ions chlorures et sodium. Le Tableav $I$ montre que les fortes corrélations sont de 0.96 entre la CE et les $\mathrm{Cl}$ et de 0.89 entre la CE et le $\mathrm{Na}^{+}$, suivis par les ions $\mathrm{Mg}^{2+}$. 
Tableau 1 : Matrice de corrélations

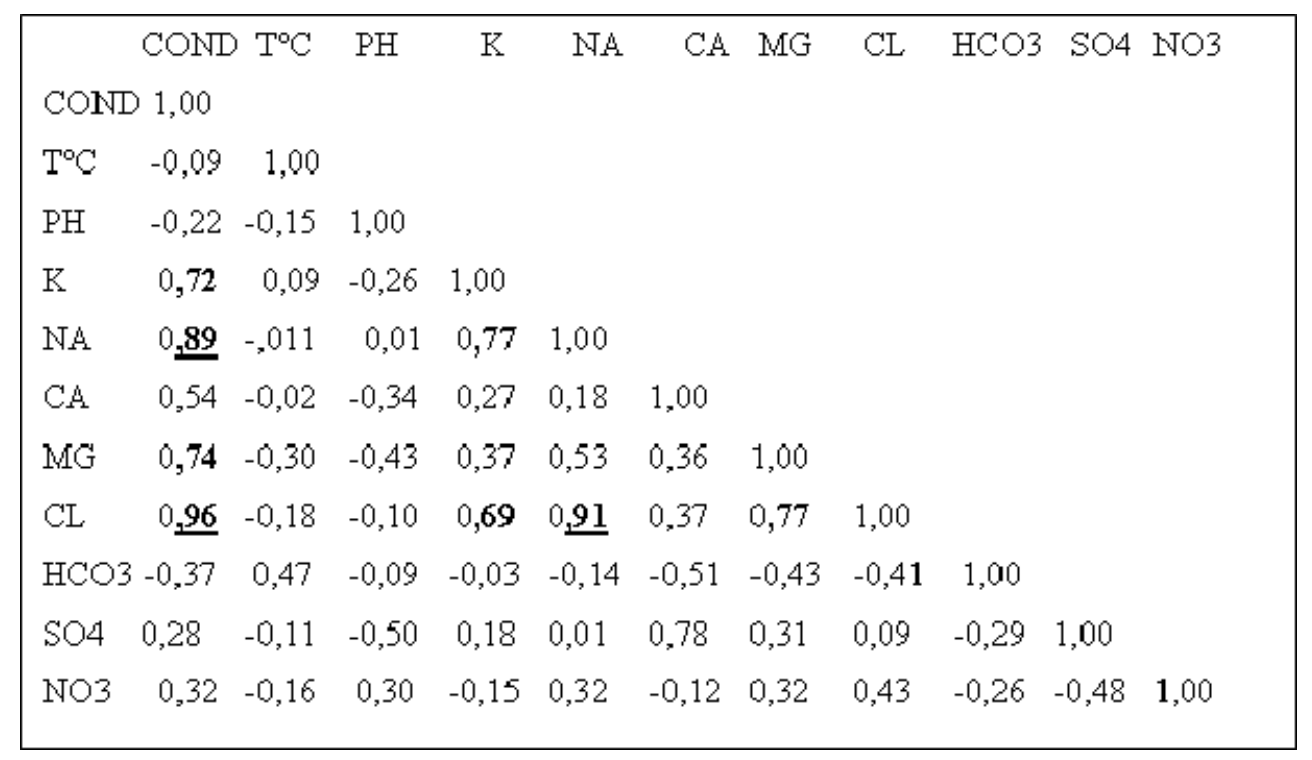

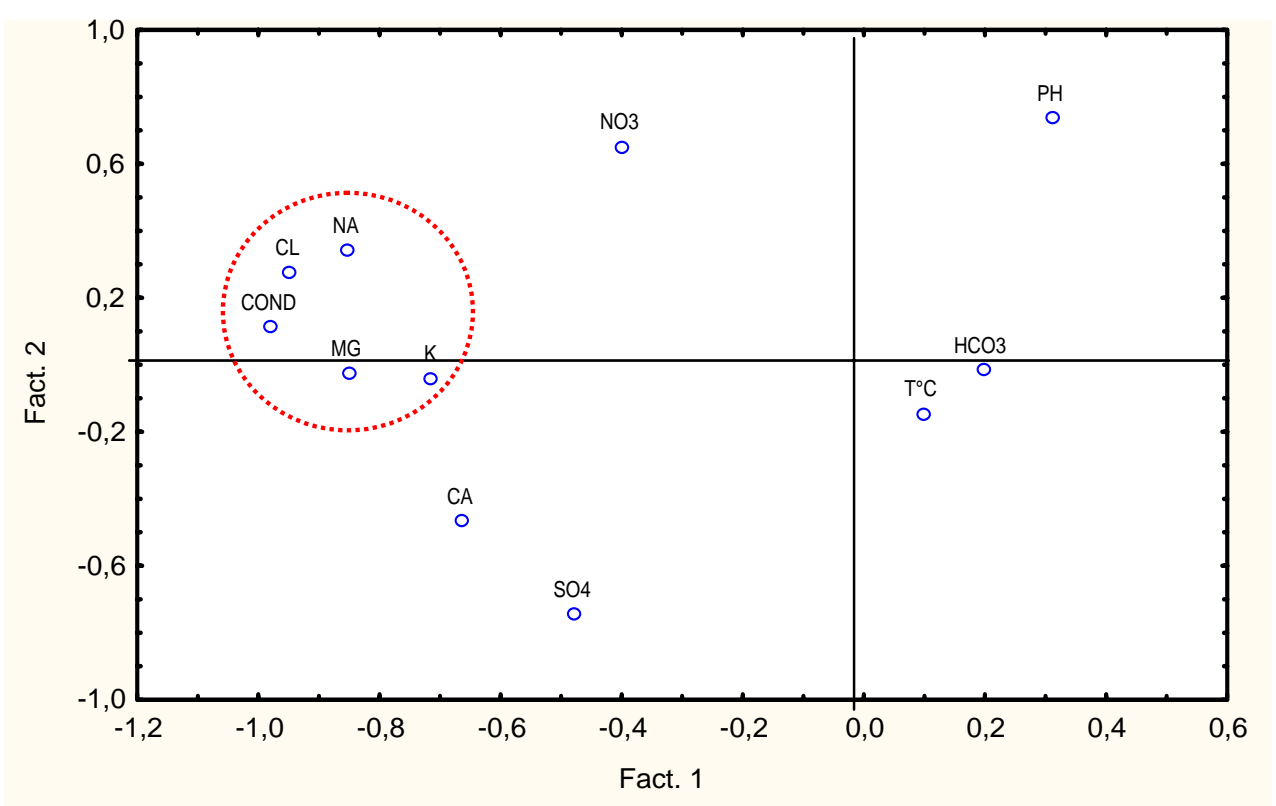

Figure 5: Analyse composante principale des données physico-chimiques 
Les cartes des chlorures et du sodium présentent de fortes similitudes avec la carte de la conductivité électrique des eaux (Figures 6 et $\Pi$, avec les mêmes secteurs à forte charge saline et le même resserrement des courbes d'isovaleurs en direction de la mer.

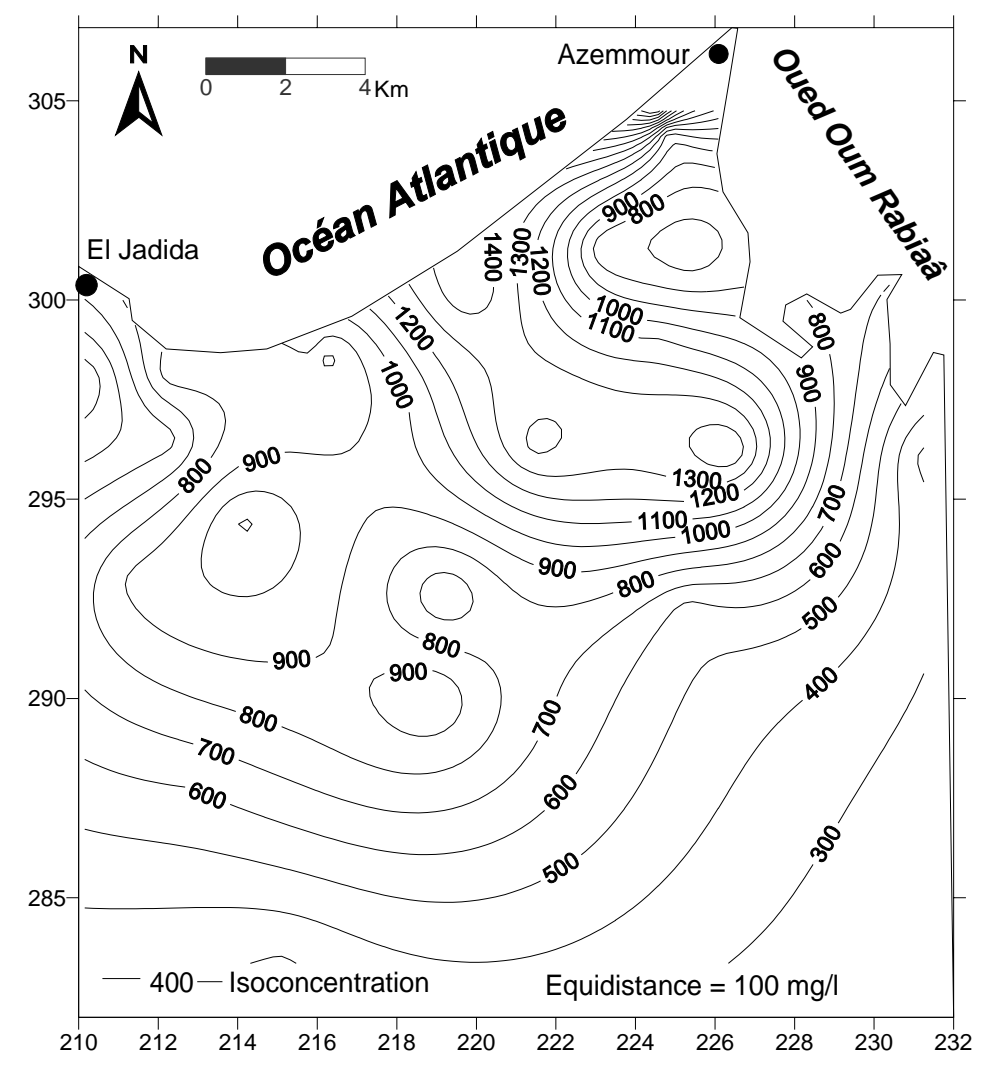

Figure 6 : Carte d'isoconcentration en Cl (Août 2005)

Ce sont toujours les secteurs côtiers et principalement ceux de la partie Nord Est qui montrent les fortes teneurs en chlorures et sodium, respectivement supérieures à 1000 et $400 \mathrm{mg} / \mathrm{l}$. Les concentrations maximales sont de $2200 \mathrm{mg} / \mathrm{l}$ en chlorures et $1050 \mathrm{mg} / \mathrm{l}$ en sodium. Comme pour les conductivités électriques, l'origine potentielle de ces deux ions dans l'eau peut être liée au phénomène d'intrusion marine surtout dans le secteur Nord Est. A cela s'ajoute les aérosols marins, l'utilisation excessive des sels en agriculture, les paléosalinités,... (es sources sont aggravées par le phénomène d'évaporation principalement dans les secteurs proches de la mer là où la nappe circule à de faibles profondeurs de la surface. 


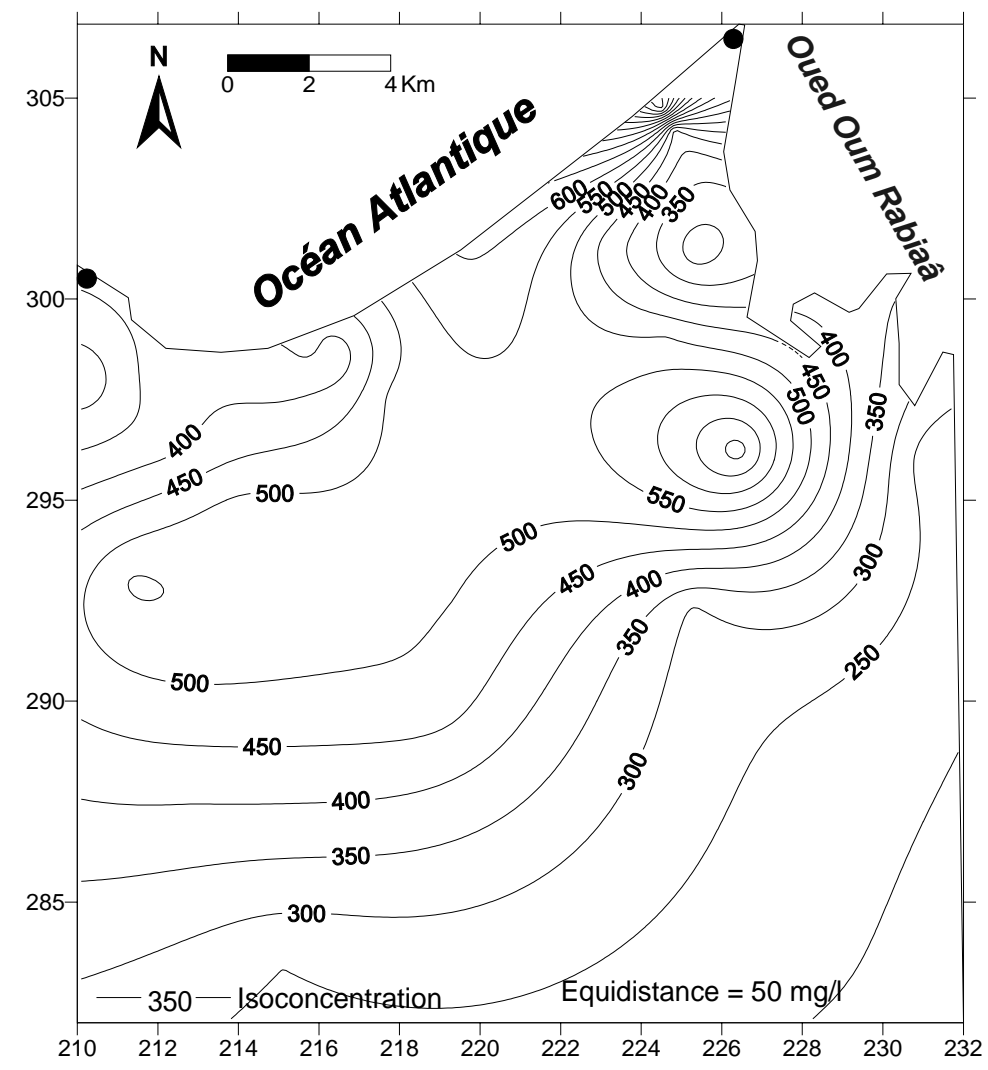

Figure 7 : Carte d'isoconcentration en $\mathrm{Na}^{+}$(Août 2005)

\section{Conclusion}

L'utilisation des outils hydrogéologiques et piézométriques et des traceurs géochimiques, a montré une meilleure adaptation de ces outils à notre système aquifère côtier, en zone agricole semi-aride. Elle a permis de vérifier nos hypothèses sur les sources potentielles des fortes salinités mesurées dans la le Sahel de l'Haouzia. Parmi ces sources potentielles, nous pouvons citer : l'invasion de la nappe par des eaux marines surtout dans les deux premiers kilomètres de la mer, les aérosols et embruns marins, les eaux de retour chargées en sels, ...

La méthode géologique et hydrogéologique a mis en évidence les principaux résultats suivants : 
- un écoulement de la nappe vers la mer (exutoire principal), à proximité de laquelle le gradient hydraulique et l'épaisseur saturée sont faibles, les réserves aquifères sont moindres, les pompages sont intensifs.

Toutes ces constations font des secteurs côtiers, des zones vulnérables à l'intrusion marine, accentuée par les sécheresses qui sévissent sur la région.

L'interprétation des résultats des différentes campagnes physico-chimiques réalisées dans le cadre de ce travail, a montré que :

* d'après les cartes et les graphiques chimiques:

- un resserrement très marqué des courbes des isovaleurs en direction de la mer, très net dans les deux premiers kilomètres surtout dans les secteurs proche du rivage.

- l'évolution des concentrations des sels dissous dans les eaux souterraines est bien corrélée avec la distance par rapport à la mer. Les forts gradients d'augmentation des concentrations sont observés dans les deux premiers kilomètres de la côte, surtout de la partie Nord Est : de 4 à $7 \mathrm{mS} / \mathrm{cm} / \mathrm{km}$ pour la conductivité électrique, de 800 à $1800 \mathrm{mg} / \mathrm{l} / \mathrm{km}$ pour les chlorures, de 350 à $950 \mathrm{mg} / \mathrm{l} / \mathrm{km}$ pour le sodium.

Combiné avec l'outil hydrogéologique et piézométrique, l'ensemble de ces résultats géochimiques plaide en faveur d'une contribution relativement importante de la source marine à la salinité des puits, principalement dans le premier kilomètre du rivage.

\section{Références}

[1] - A. El Mandour, I Contribution à l'étude hydrogéologique de la plaine de Trifa Salinisation et modélisation II, Thèse de Doctorat p, Faculté des Sciences, Oujda, Maroc (1998)

[2] - Fedrigoni, L. Krimissa, M. Zouari, K. Maliki, A. and G-M Zuppi, II Origine de la minéralisation et comportement hydrogéochimique d'une nappe phréatique soumise à des contraintes naturelles et anthropiques sévères : exemple de la nappe de Djebeniana,Tunisie II, Comptes Rendus de l'Académie des Sciences Series IIA - Earth and Planetary Science .332 (11) (2001), pp. 665-671

[3] - A .Younsi, ॥ Etude des mécanismes des intrusions marines vers le système aquifère compris entre oued Oum Er Bia et Bir Jdid province d'El Jadida : Hydrogéologie, hydrodynamique, hydrochimie et modélisation mathématique 1 , Thèse de $3^{\text {ème }}$ Cycle, Faculté des Sciences, El Jadida, Maroc (1994) 
[4] - B. Arfib and J.Ganoulis, I Modélisation physique de l'intrusion d'eau de mer dans un aquifère karstique : cas de l'Almyros d'Héraklion Crète ॥ Comptes Rendus Geosciences., 336 (11) (2004): 999-1006

[5] - R.Trabelsi, M. Zaiiri, H. Smida and H. Ben Dhia, II Salinisation des nappes côtières: cas de la nappe nord du Sahel de Sfax, Tunisie "I Comptes Rendus Geosciences., 337 (2005) : 515-524

[6] - M.Ferré and J. P. Ruhard, II Les Bassins des Abda Doukkala et du Sahel d'Azemmour à Safi. Plaines et bassins du Maroc Atlantique $॥$ (éditions du service géologique du Maroc), Ressources en Eau du Maroc Tome 2, Rabat, Maroc (1975) pp 262-298

[7] - M. Gigout, II Etude géologiques sur la Méséta marocaine occidentale ( arrière pays de Casablanca, Mazagan et Safi II. Travaux de l'institut scientifique chérifien Tome 1, Rabat, Maroc (1951) 494p.

[8] - M. Ouadia, II Les formations plioquaternaires dans le domaine mésétien occidental du Maroc entre Casablanca et Safi : géomorphologie sedimentologie Paléoenvironnements quaternaires et évolution actuelle 1 , Thèse de Doctorat, Faculté des Sciences, Rabat, Maroc (1998)

[9] - A. El Achheb, II Contribution à l'étude de la minéralisation et identification des sources de contamination des eaux souterraines, application au système aquifère du Bassin Sahel Doukkala Maroc 11 ,Thèse de Doctorat, Faculté des Sciences, El Jadida, Maroc (2002)

[10] - S. Merrad, ॥ Contribution à l'étude hydrogéologique et hydrochimique des aquifères côtiers du Sahel entre Sidi Abed et Oualidia Province d'El Jadida ॥ Mémoire du CEA, Faculté des Sciences, Rabat, Maroc (1997)

[11] - S. Khatmi, ॥ La couverture Méso-Cénozoïque des Doukkala Méséta côtière, Maroc: Stratigraphie et évolution Géodynamique II, Thèse de Doctorat, Faculté des Sciences, El Jadida, Maroc (1999) 